\title{
Heavy alcohol drinking downregulates ALDH2 gene expression but heavy smoking up-regulates SOD2 gene expression in head and neck squamous cell carcinoma
}

\author{
Dong Jin Lee*, Hyung Min Lee, Jin Hwan Kim, II Seok Park and Young Soo Rho
}

\begin{abstract}
Background: This study aims to determine the relationship between expression levels of ALDH2 and SOD2 genes and clinical parameters such as alcohol drinking, tobacco smoking, primary site of HNSCC, and human papilloma virus (HPV) state.

Methods: Gene expression data were obtained from gene expression omnibus (GEO accession number: GSE65858). Clinical data $(N=270)$ including survival result, gender, age, TNM stage, primary site of HNSCC, HPV status, alcohol drinking, and tobacco smoking habit were analyzed according to gene expression pattern.

Results: ALDH2 gene was expressed in low levels in patients with heavy alcohol consumption. It was expressed in high $(p=0.01)$ levels in patients with no or light alcohol consumption. ALDH2 gene was also expressed in low levels in patients with oral cavity cancers or hypopharynx cancers. However, ALDH2 gene was expressed in high ( $p=0.03$ ) levels in patients with oropharyngeal cancers or laryngeal cancers. HPV-positive patients were found to have high ( $p=0.02$ ) expression levels of ALDH2. SOD2 gene was expressed in high $(p=0.005)$ levels in patients who had greater mean pack-year of tobacco smoking. Based on log rank test, the group of patients with high expression of ALDH2 showed better $(p=0.002)$ clinical results than those with low expression of ALDH2. Difference of survival results between ALDH2 high-expressed group and ALDH2 low-expressed group was validated in another cohort (GSE39368, N = 138).

Conclusions: Heavy alcohol drinking downregulates ALDH2 gene expression level. Heavy smoking up-regulates SOD2 gene expression level in patients with head and neck squamous cell carcinoma. The group of patients with low expression levels of ALDH2 showed significantly poorer survival results compared to those with high expression levels of ALDH2.
\end{abstract}

Keywords: Microarray, ALDH2, SOD2, Expression, Alcohol, Smoking, Survival, HNSCC

\section{Background}

Head and neck squamous cell carcinoma (HNSCC), the fifth most common type of cancer, accounts for 600,000 newly diagnosed cancer cases with 300,000 deaths worldwide every year [1]. Despite novel surgical and pharmaceutical treatment methods developed for HNSCC, its survival rate has not been improved over the last two decades [2]. It is widely accepted that HNSCC is

\footnotetext{
* Correspondence: leesurgery@gmail.com

Department of Otolrayngology-Head and Neck Surgery, Kangnam Sacred

Heart Hospital, Hallym University College of Medicine, Seoul, South Korea
}

strongly associated with alcohol drinking and tobacco smoking [3]. It has been estimated that $75 \%$ of HNSCC cases in the USA are caused by alcohol drinking and tobacco smoking [4].

In general, the metabolism of ethanol, the major component of alcoholic beverages, consists of two steps [5]. First, alcohol dehydrogenase (ADH) enzyme converts ethanol to acetaldehyde. Second, aldehyde dehydrogenase (ALDH) converts acetaldehyde to acetate. Acetaldehyde is a toxic material that has been established as a strong carcinogen 
[6]. The second oxidation step is largely dependent on ALDH2 enzyme encoded by ALDH2 gene.

As for smoking, tobacco smoke produces a variety of free radicals, reactive oxygen species (ROS), and reactive nitrogen species (RNS). They will produce superoxide, hydrogen peroxide, and nitric oxide which can cause oxidative damage and oxidative stress to cells [7]. Superoxide dismutases (SODs) are enzymes that can catalyze the dismutation of superoxide into oxygen or hydrogen peroxide [8]. There are three distinctive SODs: copper/ zinc SOD $(\mathrm{Cu} / \mathrm{ZnSOD}$ or SOD1), manganese SOD (MnSOD or SOD2), and extracellular SOD (ecSOD or SOD3, and $\mathrm{Cu} / \mathrm{ZnSOD}$ ) [9]. Among them, SOD2 is localized at the mitochondria. It acts as an endogenous antioxidant enzyme that limits the oxidative burden [10]. SOD2 can convert superoxide into hydrogen peroxide and oxygen in the mitochondria. It plays a major role in protecting cells from oxidative damage [11].

A number of studies have demonstrated the correlation between ALDH2 gene or SOD2 gene polymorphisms and susceptibility to HNSCC [12-17]. However, the expression pattern of these two genes in HNSCC patients in terms of the amount of alcohol drinking and tobacco smoking has not been reported.

Therefore, the objectives of this study were to analyze the expression pattern of ALDH2 and SOD2 genes using available microarray data (GSE65858 and GSE39368) [18] and determine the relationship between the expression level of these two genes and clinical parameters such as alcohol drinking, tobacco smoking, primary site of HNSCC, and human papilloma virus (HPV) state.

\section{Methods}

Gene expression data and clinical information

Gene expression data (mRNA-seq) and clinical data of 270 head and neck squamous cell carcinomas were obtained from gene expression omnibus (GEO) (http:// www.ncbi.nlm.nih.gov/geo/, accession number:GSE65858) [18]. Clinical data included survival data, gender, age, TNM stage, primary site and HPV status, alcohol, and smoking habit. Amount of alcohol consumption is divided into four groups: non, light 1-30 g/day, moderate 31$60 \mathrm{~g} /$ day, heavy $>60 \mathrm{~g} /$ day (Table 1 ).

\section{Gene selection and unsupervised clustering}

We selected ADH1B, ADH1C, and ALDH2 as alcoholrelated genes and SOD1, SOD2, and SOD3 as smokingrelated genes. The BRB-ArrayTools software program (http://linus.nci.nih.gov/BRB-ArrayTools.html) was used to analyze gene expression data [19]. After gene expression data were gene-median centered, we performed unsupervised clustering of these three genes in 270 HNSCCs cases. A heatmap was generated using the Cluster and TreeView software programs [20].

\section{Statistical analysis}

All statistical analyses were performed in the $\mathrm{R}$ language (http://www.r-project.org).

Comparing mean age between groups was performed using paired $t$-test. Qui-square test were performed for all other statistical analysis. The $p$ value $<0.05$ was considered as a significant difference.

Table 1 Statistical analysis of clinical variables according to ALDH2 and SOD2 expression level

\begin{tabular}{|c|c|c|c|c|c|c|c|}
\hline & & ALDH2 high & ALDH2 low & $p$ value & SOD2 high & SOD2 low & $p$ value \\
\hline Age & Mean & 60.3 & 59.8 & 0.74 & 60.3 & 59.7 & 0.65 \\
\hline \multirow[t]{2}{*}{ Gender } & Male & 123 & 100 & 0.50 & 135 & 88 & 0.44 \\
\hline & Female & 29 & 18 & & 25 & 22 & \\
\hline Smoking & Mean pack-year & 29.4 & 32.6 & 0.28 & 34.3 & 25.9 & $* 0.005$ \\
\hline \multirow[t]{4}{*}{ Alcohol amount } & Heavy & 44 & 48 & ${ }^{*} 0.01$ & 16 & 15 & 0.15 \\
\hline & Moderate & 30 & 33 & & 43 & 41 & \\
\hline & Light & 57 & 27 & & 41 & 22 & \\
\hline & None & 21 & 10 & & 60 & 32 & \\
\hline \multirow[t]{2}{*}{ Stage } & $|-| \mid$ & 35 & 20 & 0.28 & 36 & 19 & 0.37 \\
\hline & III-IV & 117 & 98 & & 124 & 91 & \\
\hline \multirow[t]{4}{*}{ Primary site } & Oral cavity & 37 & 46 & ${ }^{*} 0.03$ & 47 & 36 & 0.94 \\
\hline & Oropharynx & 65 & 37 & & 62 & 40 & \\
\hline & Larynx & 30 & 18 & & 29 & 19 & \\
\hline & Hypopharynx & 16 & 17 & & 20 & 13 & \\
\hline \multirow[t]{2}{*}{ HPV } & Positive & 42 & 18 & ${ }^{*} 0.02$ & 34 & 26 & 0.77 \\
\hline & Negative & 109 & 100 & & 125 & 84 & \\
\hline
\end{tabular}

${ }^{*}$ means statistically significant $p$ value 
Survival analysis

The association of each group with overall survival was evaluated using Kaplan-Meier plots and log rank test. Overall survival was defined as the time from surgery to death. Data were censored when a patient was alive without recurrence at last follow-up. The $p$ value $<0.05$ was considered as a significant difference.
Significant canonical signaling pathways enriched in each group

Pathway analysis was carried by using Ingenuity Pathways Analysis (Ingenuity, Redwood City, CA), and genes from the dataset that were associated with a canonical pathway in the Ingenuity Pathways Knowledge Base were considered for the analysis.
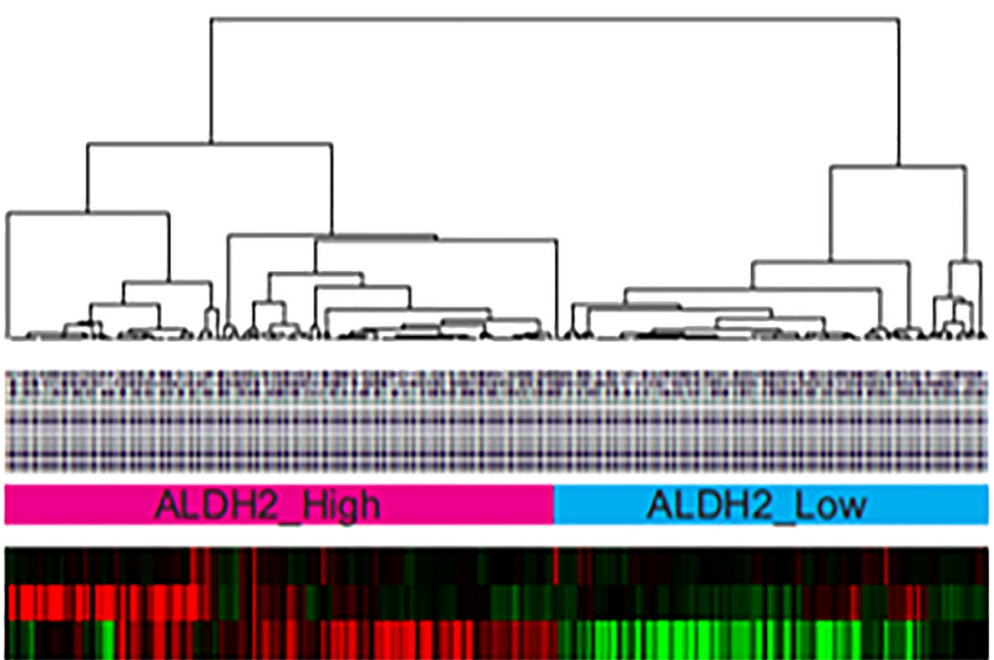

ADH1B

ADH $1 \mathrm{C}$

ALDH2

A

EXP

Low
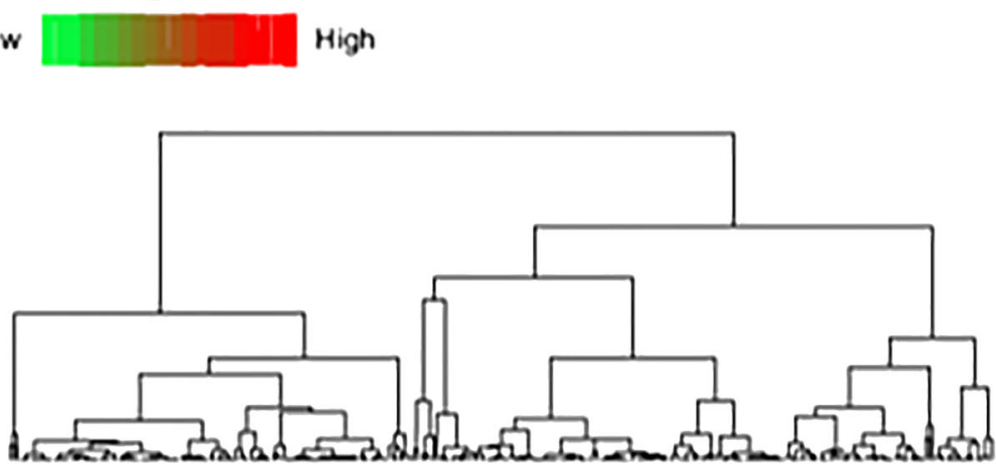

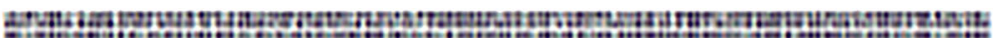

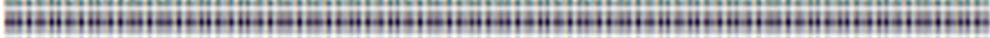

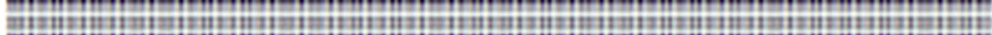

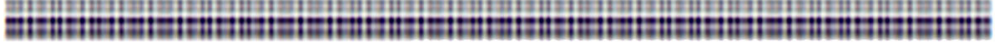

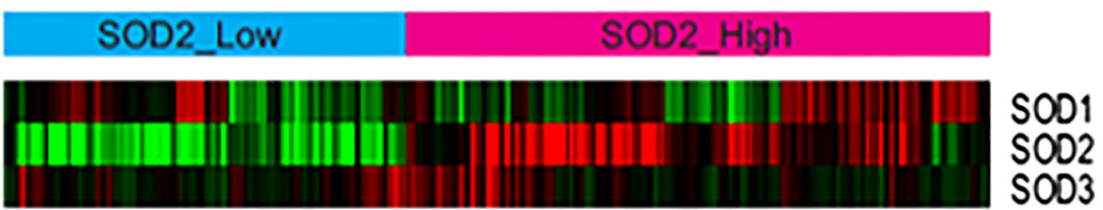

B

EXP

Low

Hgh

Fig. 1 Unsupervised clustering of 270 cases HNSCCS in GSE65858 according to aldehyde dehydrogenase (ALDH) and superoxide dismutase (SOD) genes reveals 2 molecular subtypes-ALDH2_expression_High vs ALDH2_expression_Low (a), and SOD2_expression_High vs SOD2_expression_Low (b). The data are given in matrix format. The color red or green in cell reflects relative high or low expression levels, respectively, as indicated in scale bar 
Validation in another cohort

We applied this finding to another validation cohort (GSE39368, $N=138$ ) to show the robustness of this survival difference between ALDH2 high-expressed group and ALDH2 low-expressed group.

\section{Results}

\section{Unsupervised clustering and boxplot}

We performed hierarchical clustering in an unsupervised and unbiased manner using cluster 3.0. We found two expression subtypes in alcohol-related genes and smoking-related genes (Fig. 1). For ALDH2 genes, two groups (ALDH2 high-expressed group vs. ALDH2 lowexpressed group) were obtained (Fig. 1a). For SOD2 genes, two groups (SOD2 high-expressed group vs. SOD2 low-expressed group) were also obtained (Fig. 1b). Compared to ALDH2 and SOD2 genes, other genes were not divided into two groups (Fig. 1). The difference in expression level between the two groups was determined by boxplot analysis (Fig. 2).

\section{Statistical analysis for clinical variables}

Results of statistical analysis for clinical variables including age, gender, smoking state, alcohol habit, stage, primary site, and HPV state of 270 HNSCC cases are summarized in Table 1. ALDH2 gene was expressed at low levels in patients with heavy alcohol consumption. However, it was expressed at high levels in patients with no or light alcohol consumption. ALDH2 gene was also expressed at low levels in patients with oral cavity cancers or hypopharynx cancers. However, it was expressed at high levels in patients with oropharyngeal cancers or laryngeal cancers (Table 1). HPV positive patients were found to have high expression levels of ALDH2. They were found in the ALDH2 high-expressed group. In contrast, SOD2 gene was expressed at high levels in patients who had greater mean pack-year of tobacco smoking. There was no statistically significant difference in other variables between the SOD2 high-expression and the SOD2 low-expression group (Table 1).

\section{Survival analysis}

When we compared the KM survival curve and log rank test for each high- and low-expressed group, ALDH2 high-expression group showed better survival results compared to the ALDH2 low-expression group (Fig. 3a). However, the survival results of SOD2 high-expression group were not significantly different from those of SOD2 low-expression group (Fig. 3b).

\section{Significant canonical signaling pathways enriched in each group}

Pathway analysis revealed that ALDH2 high-expression group was enriched in canonical pathways such as Cdc42 signaling, lymphotoxin $\beta$ receptor signaling, RhoA signaling, and intrinsic prothrombin activation pathway (Table 2). SOD2 high-expression group was also enriched in canonical pathways such as TREM1 signaling, PPAR signaling, PI3K/AKT signaling, and protein kinase A signaling pathway (Table 2).

\section{Validation in another cohort}

We applied this finding to another validation cohort (GSE39368, $N=138$ ) to show the robustness of this survival difference between ALDH2 high-expressed group and ALDH2 low-expressed group. For ALDH2 genes, two groups (ALDH2 high-expressed group vs. ALDH2 low-expressed group) were obtained (Fig. 4a). For SOD2 genes, two groups (SOD2 high-expressed group vs. SOD2 low-expressed group) were also obtained (Fig. 4b). When we compared the KM survival curve and log rank test for each high- and low-expressed group, ALDH2 high-
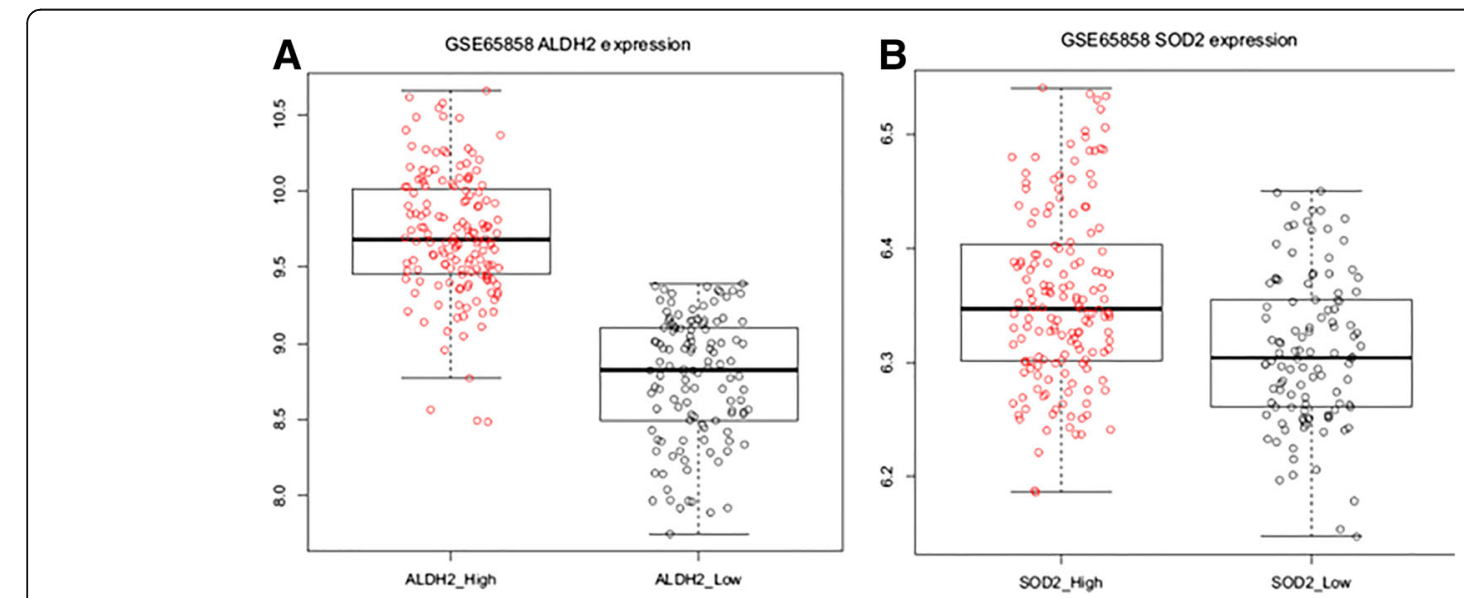

Fig. 2 Boxplots shows the difference of gene expression level between two groups. a The difference of ALDH2 gene expression level. b The difference of SOD2 gene expression level 

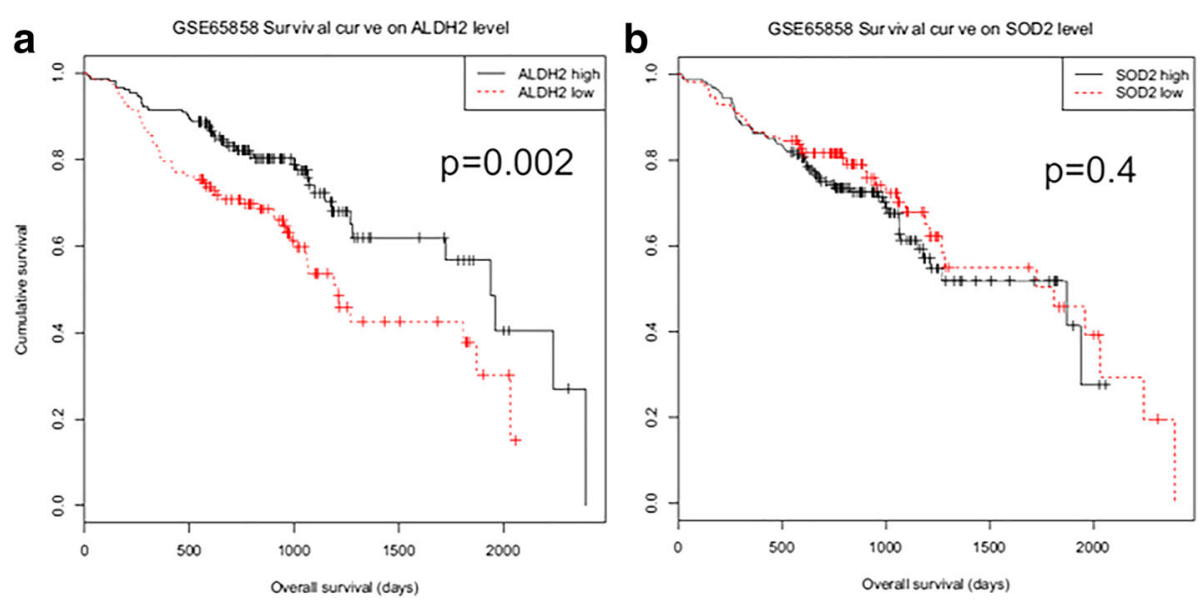

Fig. 3 Kaplan-Meier survival curve according to ALDH2 and SOD2 expression level. ALDH2_high group showed better prognosis when compared with ALDH2_low group $(p=0.002$, (a)). In contrast, SOD2 gene expression level did not show any survival difference $(p=0.4$, (b))

expression group showed better survival results compared to the ALDH2 low-expression group (Fig. 5a, $p=0.03$ ). However, the survival results of SOD2 high-expression group were not significantly different from those of SOD2 low-expression group (Fig. 5b, $p=0.4$ ).

\section{Discussion}

In this study, we evaluated the association between expression levels of alcohol metabolism genes or ROS metabolism genes and the amount of alcohol drinking or tobacco smoking. We also determined the association of gene expression patterns with clinical variables such as primary site, HPV state, stage, and survival result. Among alcohol metabolism genes, ALDH2 gene expression pattern divided the microarray data into two groups: ALDH2 high-expression group $(N=152)$ and ALDH2 low-expression group $(N=118)$ (Fig. 1a). Interestingly, heavy alcohol consumption group showed low expression levels of ALDH2 with poor overall survival results (Table 1 and Fig. 3a). Microarray data $(N=270)$ were also divided into two groups by SOD2 expression pattern (Fig. 1b). However, in contrast to the relationship between ALDH2 expression and alcohol consumption amount, heavy smoking group showed high SOD2 gene expression (Table 1). Interestingly, there was no correlation between SOD2 expression level and survival results (Fig. 3b).

Alcohol drinking and tobacco smoking are significant risk factors for head and neck cancer [3]. In general, alcohol metabolism involves two steps. First, alcohol is

Table 2 Altered canonical pathways in each group

\begin{tabular}{|c|c|c|c|c|}
\hline & -Log ( $p$ value) & Ratio & z-score & Molecules \\
\hline \multicolumn{5}{|l|}{$\begin{array}{l}\text { Altered pathways in ALDH2 high-expressed } \\
\text { group }\end{array}$} \\
\hline $\begin{array}{l}\text { Role of NFAT in regulation of the } \\
\text { immune response }\end{array}$ & 4.82E00 & $2.34 \mathrm{E}-01$ & 3.157 & $\mathrm{BLNK}, \mathrm{CD} 247, \mathrm{RAF} 1, \mathrm{HLA}-\mathrm{DOA}, \mathrm{CD} 3 \mathrm{E}, \mathrm{CD} 4, \mathrm{GNA} 11$,FCER1A,HLA-DQA1 \\
\hline Cdc42 signaling & $3 \mathrm{E} 00$ & $2.04 \mathrm{E}-01$ & -2.828 & CD247,RAF1,HLA-DOA,MAP3K11,CD3E,ARPC1B,HLA-DQA1,CDC42EP2 \\
\hline Lymphotoxin $\beta$ receptor signaling & $1.32 \mathrm{E} 00$ & $2.04 \mathrm{E}-01$ & 2.714 & IKBKB,MAP3K14,VCAM1,AKT1,CASP9,LTA,PIK3CG,LTB,IKBKE,PIK3CB \\
\hline RhoA signaling & 4.37E-01 & $1.31 \mathrm{E}-01$ & -2.309 & NEDD4,CFL1,NRP2,ARPC1B,ANLN,GNA12,RTKN \\
\hline Intrinsic prothrombin activation pathway & $5.98 \mathrm{E}-01$ & $1.72 \mathrm{E}-01$ & -2.236 & COL1A2,COL1A1,COL5A3,COL10A1,COL3A1 \\
\hline \multicolumn{5}{|l|}{$\begin{array}{l}\text { Altered pathways in SOD2 high-expressed } \\
\text { group }\end{array}$} \\
\hline TREM1 signaling & $8.12 \mathrm{E} 00$ & 2.27E-01 & 3.638 & RELA,CXCL8,TREM1,NLRP3,PLCG1,IL6,CCL3,NOD2,CCL2,CASP1 \\
\hline PPAR signaling & 3.95E00 & $1.4 \mathrm{E}-01$ & -3.606 & RELA,IL36G,IL1A,NRAS,RRAS2,NFKBIA,HSP90AB1,PPARD,IL1B \\
\hline PI3K/AKT signaling & $3.78 \mathrm{E} 00$ & $1.22 \mathrm{E}-01$ & 2.324 & TSC1,RELA,NRAS,YWHAH,PIK3R1,MAP3K5,ITGA3,NFKBIA,RRAS2 \\
\hline Protein kinase A signaling & 3.16E00 & 7.77E-02 & -2.294 & RELA,YWHAH,PDE7A,PTPN2,ANAPC10,PTPN12,GNG7,PHKA2,GNB3 \\
\hline TNFR1 signaling & 1.83E00 & $1.22 \mathrm{E}-01$ & 2.236 & RELA,NFKBIA,TNFAIP3,NFKBIB,TNF,CASP7 \\
\hline IL-1 signaling & 3.45E00 & $1.32 \mathrm{E}-01$ & 2.121 & RELA,IL1A,GNB3,NFKBIA,GNA15,GNB2,ADCY6,IRAK3,NFKBIB,GNG5 \\
\hline
\end{tabular}



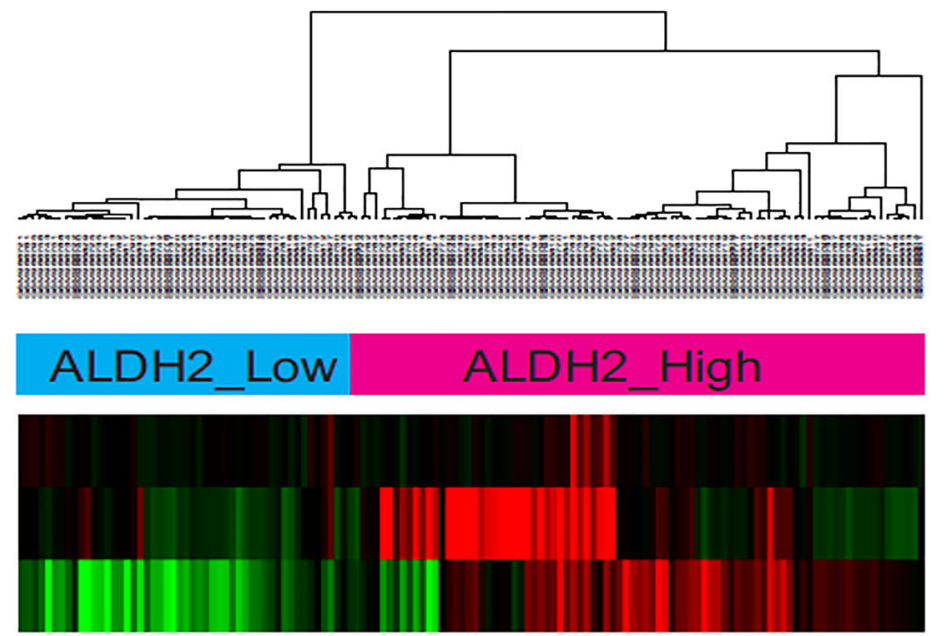

a
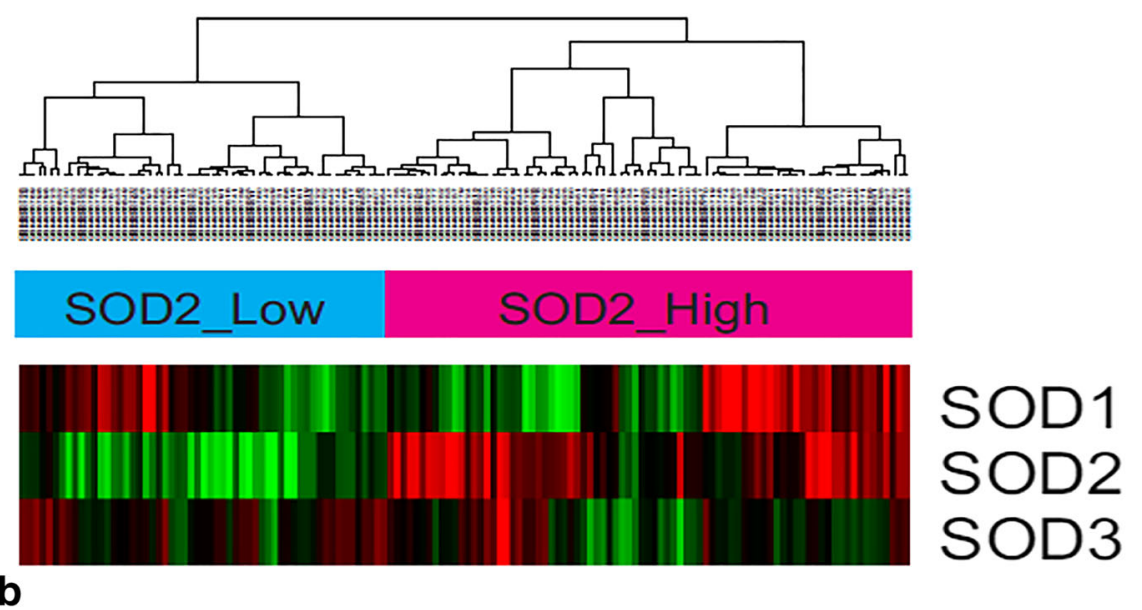

Fig. 4 Unsupervised clustering of 183 cases HNSCCs in GSE39368 according to aldehyde dehydrogenase (ALDH) and superoxide dismutase (SOD) genes reveals two molecular subtypes-ALDH2_expression_High vs ALDH2_expression_Low (a), and SOD2_expression_High vs SOD2_expression_Low (b)

converted into acetaldehyde by ADH. Second, acetaldehyde is oxidized into nontoxic acetate by ALDH [5]. Among all classes of $\mathrm{ADH}$ and ALDH isoenzymes, ADH1B, ADH1C, and ALDH2 are the main ethanolmetabolizing enzymes [21, 22]. Therefore, we used these three genes to analyze the expression pattern of alcohol metabolism genes. As for ROS metabolism genes, SODs are enzymes that catalyze the removal of superoxide free radicals [8]. SODs are present in all aerobic living cells. This is probably because $\mathrm{O}_{2}^{-}$is a common product of oxygen metabolic reactions. In mammals, there are three distinctive SODs: SOD1 (Cu/ZnSOD), SOD2 (MnSOD), and SOD3 (extracellular SOD). SOD1 is the major intracellular form of SODs. It is primarily cytosolic [23]. In contrast, SOD2 is exclusively localized in the mitochondrial matrix (MM) [23], while SOD3 is the secreted form mainly associated with the extracellular matrix of different tissues [24]. In this study, we used these three superoxide dismutases genes to analyze the expression patterns of ROS metabolism genes. Among these three alcohol-related genes, only ALDH2 gene showed distinguished expression pattern. Similarly, among the three smoking-related genes, only SOD2 gene showed distinguished expression subtype.

Several studies have demonstrated the correlation between ALDH2 gene or SOD2 gene polymorphisms and susceptibility to cancer development. Zhao et al. have reported that ALDH2 Glu504Lys SNP is a potential candidate genetic risk factor for a variety of chronic diseases such as cardiovascular disease, cancer, and late-onset Alzheimer's disease [12]. Chung et al. have suggested that minor alleles of ADH1B (rs1229984) and ALDH2 


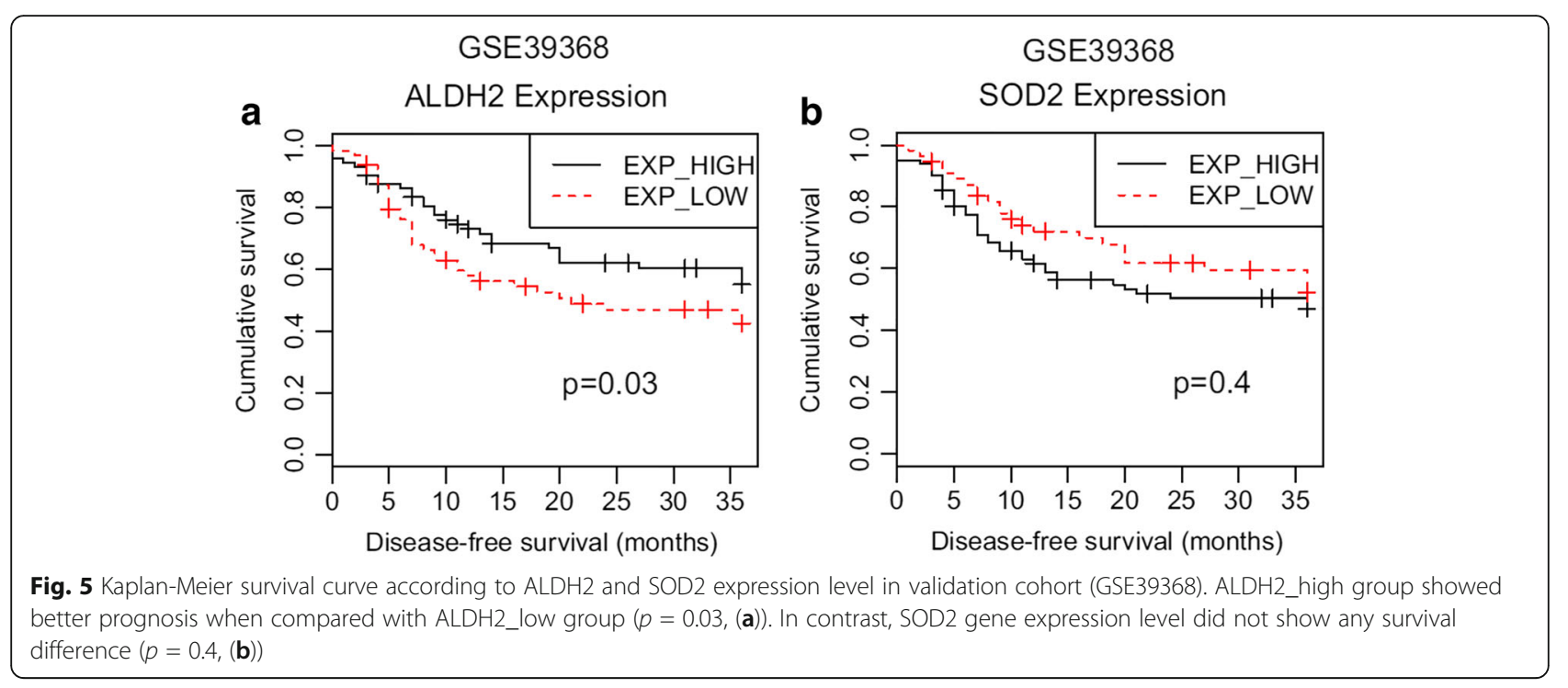

(rs671) not only are associated with the risk of upper aerodigestive tract cancer, but also can potentiate the carcinogenic effects of alcohol [13]. Hidaka et al. have reported that ALDH2 AA allele carriers who drink > $150 \mathrm{~g} /$ week have an increased risk of cancer development compared to GG genotype carriers who drink 0 to $<150 \mathrm{~g} /$ week [15]. As for SOD2, Han et al. have reported that polymorphism of SOD2 T5482C might be closely associated with increased susceptibility to the development and differentiation of gastric cancer in Korean population [16]. All these SNP studies have emphasized the important roles of ALDH2 and SOD2 in alcohol drinking and smokinginduced cancer development. However, up to date, no study has reported the expression levels of these genes, especially using microarray mRNA-Seq data. In this study, we used mRNA-seq data and evaluated the relationship between expression levels of ALDH2 and SOD2 genes and the amount of alcohol drinking or smoking consumption.

In statistical analysis, the amount of alcohol consumption, primary site, and HPV state showed statistically significant difference between ALDH2 high-expression group and ALDH2 low-expression group (Table 1). Patients with heavy alcohol consumption showed low ALDH2 expression levels, while patients with no or light alcohol consumption showed high ALDH2 expression levels (Table 1). There are two possible hypotheses to explain such results. First, cumulative acetaldehyde itself might have downregulated the expression of ALDH2 gene directly or indirectly using other upstream or downstream molecules. Second, the liver functions of heavy alcoholics usually are not so good. Their anabolic functions such as mRNA synthesis and protein synthesis are usually decreased. The finding that patients in the heavy smoking group showed high SOD2 expression in this study supports the second hypothesis.
In this study, we used Ingenuity Pathway Analysis (IPA) to investigate up-regulated and downregulated pathways in ALDH2 high-expression group and SOD2 high-expression group. Intrinsic prothrombin activation pathway is up-regulated in ALDH2 low-expression group (z-score 2.236, Table 2). Garcia-Banuelos et al. have investigated the relationship between ALDH2 SNP and liver cirrhosis [25]. Heavy alcohol drinking can cause liver damage and activate intrinsic prothrombin activation pathway, especially in the ALDH2 lowexpression group. Chao et al. have reported the interplay between superoxide dismutase genes and PPAR signaling [26]. In our study, the SOD2 high-expression group showed decreased PPAR signaling pathway (Table 2).

This study has some limitations. First, this study is a retrospective study. Second, no data about liver function state of patients enrolled in this study were available. Comparing liver functions between heavy alcoholics and those with no or light alcohol consumption is needed as we mentioned above. A prospective and functional study is needed to provide more precise information about the role and prognostic aspect of ALDH2 and SOD2 genes in head and neck cancer.

\section{Conclusions}

In conclusion, this is the first study describing the relationship between the amount of alcohol drinking or smoking consumption and alcohol or ROS metabolizing gene expression level. In this study, heavy alcohol drinking downregulated ALDH2 gene expression while heavy smoking up-regulated SOD2 gene expression in head and neck squamous cell carcinoma patients $(N=270)$. This result can be used to predict the survival results of HNSCC patients, especially those heavy alcoholics with HNSCC. 


\section{Acknowledgements}

We appreciated patients and their families who generously donated their tissues to TCGA, as well as the members of TCGA who collected and disclosed valuable data.

\section{Funding}

This research was supported by Hallym University Research Fund 2013(HURF-2013-22).

\section{Availability of data and materials}

Gene expression data (mRNA-seq) and clinical data of 270 head and neck squamous cell carcinomas (HNSCCS) were obtained from gene expression omnibus (GEO) (http://www.ncbi.nlm.nih.gov/geo/, accession number: GSE65858). Also, clinical data of 183 HNSCCs were obtained from GEO (http://www.ncbi.nlm.nih.gov/geo/, accession number: GSE39368) as a validation cohort.

\section{Authors' contributions}

DJL designed this study, analyzed and interpreted TCGA data, and was a major contributor in writing the manuscript. HML gathered GEO data (GSE 65858 and GSE 39368) and analyzed these data. JHK performed TCGA data analysis. ISP performed pathway analysis using IPA (Ingenuity Pathway Analysis). YSR designed this study and performed statistical analysis. All authors read and approved the final manuscript.

\section{Ethics approval and consent to participate}

Not applicable.

\section{Consent for publication}

Not applicable.

\section{Competing interests}

The authors declare that they have no competing interests.

\section{Publisher's Note}

Springer Nature remains neutral with regard to jurisdictional claims in published maps and institutional affiliations.

\section{Received: 8 January 2017 Accepted: 13 August 2017}

Published online: 25 August 2017

\section{References}

1. Ferlay J, Soerjomataram I, Dikshit R, Eser S, Mathers C, Rebelo M, et al. Cancer incidence and mortality worldwide: sources, methods and major patterns in GLOBOCAN 2012. Int J Cancer. 2015;136:E359-86.

2. Jemal A, Bray F, Center MM, Ferlay J, Ward E, Forman D. Global cancer statistics. CA. 2011;61:69-90.

3. Secretan B, Straif K, Baan R, Grosse Y, El Ghissassi F, Bouvard V, et al. A review of human carcinogens-part E: tobacco, areca nut, alcohol, coal smoke, and salted fish. Lancet Oncol. 2009;10:1033-4.

4. Blot WJ, McLaughlin JK, Winn DM, Austin DF, Greenberg RS, Preston-Martin S, et al. Smoking and drinking in relation to oral and pharyngeal cancer. Cancer Res. 1988;48:3282-7.

5. Klyosov AA. Kinetics and specificity of human liver aldehyde dehydrogenases toward aliphatic, aromatic, and fused polycyclic aldehydes. Biochemistry. 1996:35:4457-67.

6. Humans IWGotEoCRt. Personal habits and indoor combustions. Volume 100 E. A review of human carcinogens. IARC monographs on the evaluation of carcinogenic risks to humans/World Health Organization, International Agency for Research on Cancer. 2012;100:1-538.

7. Ames BN, Gold LS. Endogenous mutagens and the causes of aging and cancer. Mutat Res. 1991;250:3-16.

8. Borgstahl GE, Parge HE, Hickey MJ, Johnson MJ, Boissinot M, Hallewell RA, et al. Human mitochondrial manganese superoxide dismutase polymorphic variant Ile58Thr reduces activity by destabilizing the tetrameric interface. Biochemistry. 1996;35:4287-97.

9. McCord JM, Fridovich I. Superoxide dismutase: the first twenty years (1968-1988). Free Radic Biol Med. 1988;5:363-9.

10. Hubackova M, Vaclavikova R, Ehrlichova M, Mrhalova M, Kodet R, Kubackova K, et al. Association of superoxide dismutases and $\mathrm{NAD}(\mathrm{P}) \mathrm{H}$ quinone oxidoreductases with prognosis of patients with breast carcinomas. Int J Cancer. 2012:130:338-48

11. Valko M, Leibfritz D, Moncol J, Cronin MT, Mazur M, Telser J. Free radicals and antioxidants in normal physiological functions and human disease. Int J Biochem Cell Biol. 2007;39:44-84.

12. Zhao Y, Wang C. Glu504Lys single nucleotide polymorphism of aldehyde dehydrogenase 2 gene and the risk of human diseases. Biomed Res Int. 2015;2015:174050.

13. Chung CS, Lee YC, Liou JM, Wang CP, Ko JY, Lee JM, et al. Tag single nucleotide polymorphisms of alcohol-metabolizing enzymes modify the risk of upper aerodigestive tract cancers: HapMap database analysis. Dis Esophagus. 2014;27:493-503.

14. Bediaga NG, Marichalar-Mendia X, Rey-Barja N, Setien-Olarra A, Gonzalez-Garcia JA de Pancorbo MM, et al. Polymorphisms in alcohol and tobacco metabolism genes in head and neck cancer in the Basque Country. Journal of oral pathology \& medicine. 2015;44:769-75.

15. Hidaka A, Sasazuki S, Matsuo K, Ito H, Sawada N, Shimazu T, et al. Genetic polymorphisms of $\mathrm{ADH} 1 \mathrm{~B}, \mathrm{ADH} 1 \mathrm{C}$ and $\mathrm{ALDH} 2$, alcohol consumption, and the risk of gastric cancer: the Japan Public Health Center-based prospective study. Carcinogenesis. 2015;36:223-31.

16. Han L, Lee SW, Yoon JH, Park YG, Choi YJ, Nam SW, et al. Association of SOD1 and SOD2 single nucleotide polymorphisms with susceptibility to gastric cancer in a Korean population. APMIS. 2013;121:246-56.

17. Yi JF, Li YM, Liu T, He WT, Li X, Zhou WC, et al. Mn-SOD and CuZn-SOD polymorphisms and interactions with risk factors in gastric cancer. World J Gastroenterol. 2010;16:4738-46.

18. Wichmann G, Rosolowski M, Krohn K, Kreuz M, Boehm A, Reiche A, et al. The role of HPV RNA transcription, immune response-related gene expression and disruptive TP53 mutations in diagnostic and prognostic profiling of head and neck cancer. Int J Cancer. 2015;137:2846-57.

19. LA SR, Li MC, Ngan M, Menenzens S, Zhao Y. Analysis of gene expression data using BRB-Array Tools. Cancer Inform. 2007;3:11-7.

20. Eisen MB, Spellman PT, Brown PO, Botstein D. Cluster analysis and display of genome-wide expression patterns. Proc Natl Acad Sci U S A. 1998;95:14863-8.

21. Ehrig T, Bosron WF, Li TK. Alcohol and aldehyde dehydrogenase. Alcohol Alcoholism. 1990;25:105-16.

22. Bosron WF, Li TK. Genetic-polymorphism of human-liver alcohol and aldehyde dehydrogenases, and their relationship to alcohol metabolism and alcoholism. Hepatology. 1986;6:502-10.

23. Slot JW, Geuze HJ, Freeman BA, Crapo JD. Intracellular localization of the copper-zinc and manganese superoxide dismutases in rat liver parenchymal cells. Laboratory Investigation; a Journal of Technical Methods and Pathology. 1986;55:363-71.

24. Marklund SL. Extracellular superoxide dismutase in human tissues and human cell lines. J Clin Invest. 1984;74:1398-403.

25. Garcia-Banuelos J, Panduro A, Gordillo-Bastidas D, Gordillo-Bastidas E, Munoz-Valle JF, Gurrola-Diaz CM, et al. Genetic polymorphisms of genes coding to alcohol-metabolizing enzymes in western Mexicans: association of CYP2E1* 2 /CYP2E1*5B allele with cirrhosis and liver function. Alcohol Clin Exp Res. 2012;36:425-31.

26. Chao CT, Chen YC, Chiang CK, Huang JW, Fang CC, Chang CC, et al. Interplay between superoxide dismutase, glutathione peroxidase, and peroxisome proliferator activated receptor gamma polymorphisms on the risk of end-stage renal disease among Han Chinese patients. Oxid Med Cell Longev. 2016;2016;8516748. doi:10.1155/2016/8516748. Epub 2016 Jan 6.

\section{Submit your next manuscript to BioMed Central and we will help you at every step:}

- We accept pre-submission inquiries

- Our selector tool helps you to find the most relevant journal

- We provide round the clock customer support

- Convenient online submission

- Thorough peer review

- Inclusion in PubMed and all major indexing services

- Maximum visibility for your research

Submit your manuscript at www.biomedcentral.com/submit
) Biomed Central 